\title{
Embolização pré operatória no tratamento de Paraganglioma abdominal: relato de caso
}

\section{Pré-operative embolization of abdominal paraganglioma - case report}

Fernando Nakamura, ACBC-SP ${ }^{1}$; Rodrigo Altenfelder Silva, TCBC-SP²; Vanessa Prado dos Santos ${ }^{3}$; Álvaro Razuk Filho, TCBC-SP4; Roberto Augusto Caffaro ${ }^{5}$

\section{INTRODUÇÃO}

$\mathrm{O}$ s paragangliomas são feocromocitomas de localização extra adrenal. São tumores de células originárias da crista neural e fazem parte do sistema neuroendócrino difuso. Estas células provém dos gânglios autônomos e devido a este fato, estão topograficamente próximas aos gânglios simpáticos, à aorta e seus principais ramos' ${ }^{1}$.

São tumores raros e podem ocorrer em diversas localizações. Na região abdominal podem atingir grandes dimensões e comprimir estruturas adjacentes, além de produzir catecolaminas causando crises hipertensivas, alterações da motilidade intestinal, infarto agudo do miocárdio e arritmias cardíacas.

Existem relatos de tumores múltiplos e sincrônicos e apesar de ser infreqüente a malignidade ${ }^{1}$, pode haver o aparecimento de metástases até dez anos após o diagnóstico do tumor primário, o que requer um período de observação prolongada².

Relatamos um caso de um paciente que apresentava sinais e sintomas de hiperestimulação adrenérgica além de uma massa em região abdominal cujo resultado anatomopatológico de uma biópsia mostrou tratar-se de paraganglioma.

\section{RELATO DO CASO}

F.L.S., masculino, 55 anos queixava-se de constipação intestinal há dois anos, cólica abdominal e perda de $10 \mathrm{~kg}$ nesse período. Concomitantemente apresentava hipertensão arterial de difícil controle, palpitações e sudorese intensa.

Ao exame físico a freqüência cardíaca era de 90 batimentos por minuto e pressão arterial de $220 \times 120$ mmhg. O abdome era indolor, flácido e sem massas percutíveis ou palpáveis.

Realizou uma tomografia que mostrou em retroperitônio, formação expansiva heterogênea com intenso realce ao meio de contraste, medindo $8,7 \times 6,5 \times 7,0$ $\mathrm{cm}$, envolvendo a aorta e a cava, retificando a parede anterior da veia cava inferior, sem plano de clivagem com a terceira porção do duodeno.

Foi submetido a uma laparotomia na qual foi realizada apenas a biópsia da lesão em função de dúvida diagnóstica e do envolvimento dos grandes vasos, cujo resultado anatomopatológico foi de paraganglioma.

Em função deste diagnóstico optou-se por nova laparotomia, mas devido ao tamanho, localização e da vascularização do tumor, realizamos no pré-operatório uma aortografia que permitiu a identificação e embolização dos seus ramos nutrientes com partículas de polyvinyl alcohol (PVA), obtendo-se diminuição da vascularização tumoral (Figura 1).

Quatro dias após, foi realizada a laparotomia exploradora, encontrando-se uma massa firmemente aderida ao duodeno, aorta infra-renal e veia cava inferior. Após dissecção cuidadosa no plano sub-adventicial dos grandes vasos conseguiu-se a ligadura e secção dos ramos arteriais nutrientes advindos da aorta infra-renal e de ramos venosos que drenavam diretamente para a veia cava inferior (Figura 2).

Foi realizado o controle pressórico no intra-operatório com o uso de nitroprussiato endovenoso até a ligadura dos ramos venosos. Assim que tais ramos foram ligados e seccionados houve hipotensão, suspendendo-se de imediato a medicação hipotensora.

O paciente evoluiu bem, normalizando os níveis pressóricos sem uso de medicações anti-hipertensivas, recebendo alta hospitalar no quinto dia de pós-operatório.

\section{DISCUSSÃO}

Os paragangliomas são tumores raros, sendo encontrados na literatura diferentes modalidades de tratamento, destacando-se a cirurgia, radioterapia, quimioterapia e radiocirurgia esterotáxica ${ }^{3}$. A indicação da angiografia pré-operatória com embolização tumoral com partículas de poyvinyl alcohol (PVA) tem sido motivo de discussão.

Trabalho realizado no Setor de Cirurgia Endovascular do Departamento de Cirurgia da Santa Casa de São Paulo- São Paulo - SP.

1. Ex- Residente do Departamento de Cirurgia da Faculdade Ciências Médicas da Santa Casa de São Paulo- São Paulo - SP; 2. Professor Adjunto; Chefe do Grupo de Vias Biliares e Pâncreas do Departamento de Cirurgia da Santa Casa de São Paulo - São Paulo - SP; 3 . ExProfessora Instrutora da Disciplina de Cirurgia Vascular do Departamento de Cirurgia da Santa Casa de São Paulo- São Paulo - SP; 4.Professor Assistente da Disciplina de Cirurgia Vascular do Departamento de Cirurgia da Santa Casa de São Paulo - São Paulo - SP; 5. Professor Adjunto; Chefe da Disciplina de Cirurgia Vascular do Departamento de Cirurgia da Santa Casa de São Paulo- São Paulo - SP. 


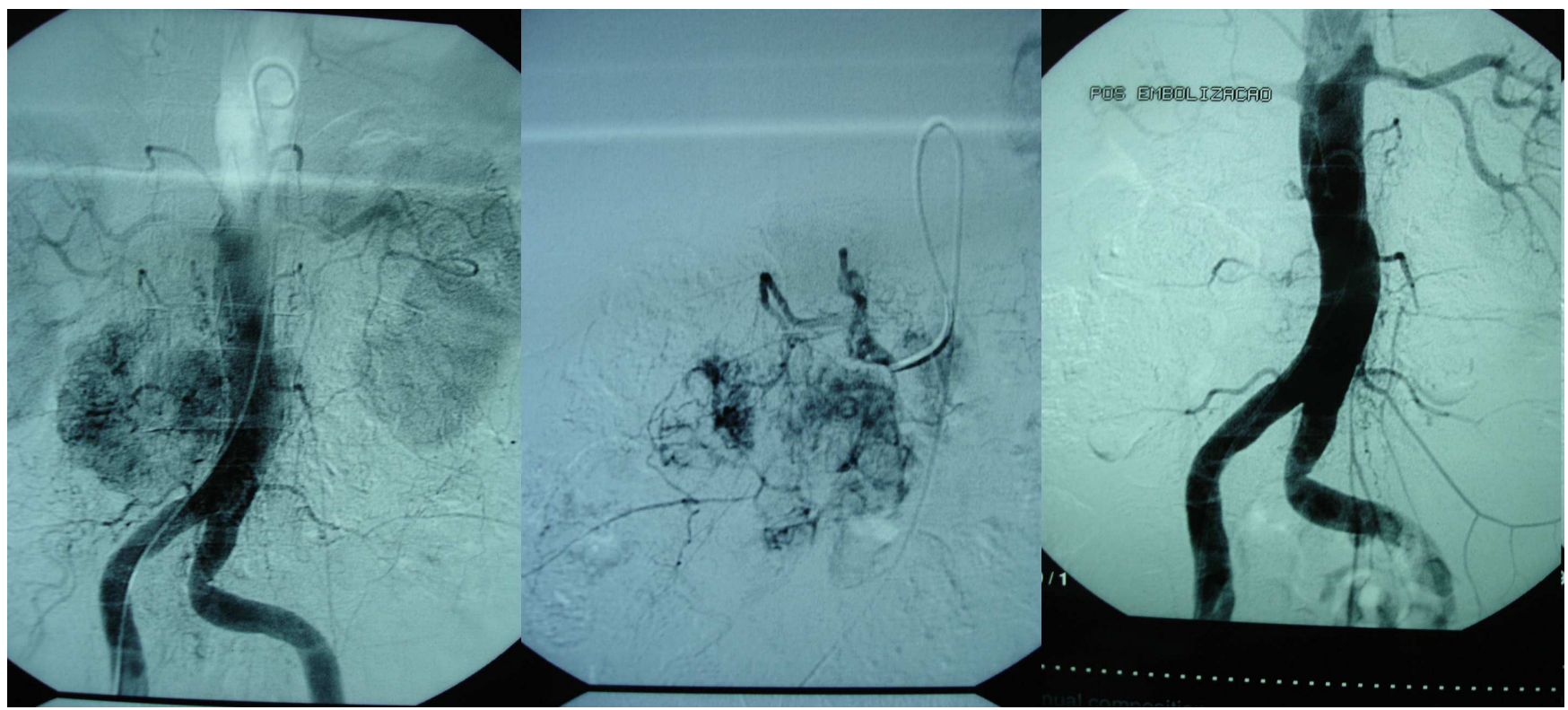

Figura 1 - Angiografia digital pré embolização, durante a cateterização seletiva para embolização e aspecto pós-embolozação.

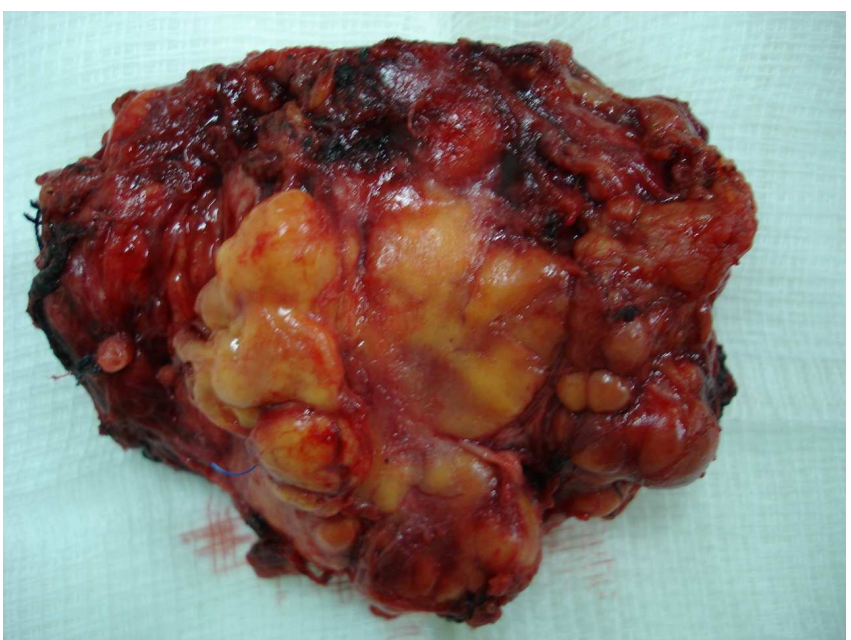

Figura 2 - Detalhe da peça cirúrgica, após ressecção do tumor.

A maioria dos trabalhos ${ }^{3}$ mostra casuísticas de paragangliomas cervicais tratados com embolização préoperatória, porém na literatura revisada não foram encontrados artigos em que tal método tenha sido aplicado em paragangliomas abdominais.

Estudos favoráveis a embolização ${ }^{4,5}$, mostraram uma significante redução do sangramento durante o ato cirúrgico, além de uma diminuição do tempo operatório.

No entanto, Boedeker et al. ${ }^{3}$, comparando a embolização pré-operatória com doentes operados sem a realização da mesma, mostraram resultados estatisticamente não significativos em relação ao tempo cirúrgico, risco de sangramento e tempo de internação.

No caso apresentado, o estudo angiográfico préoperatório foi capaz de avaliar adequadamente o suprimento arterial do tumor e suas relações com estruturas vasculares adjacentes. A embolização permitiu uma ressecção mais segura, embora nos pareça que a intervenção cirúrgica deva ser realizada mais precocemente.

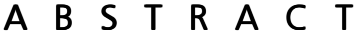

Paragangliomas is a pheochromocytoma of extra adrenal localization. The case report is a male, 55 years old who presented symptoms of adrenergic hyperstimulation associated to an abdominal mass diagnosed as paraganglioma by a biopsy. Because of its size, localization and vascularization, an aortography with embolization of the nutrient branches of the tumor was done preoperatively. Four days later, a surgical ressection was performed, and the tumor was adhered to the duodenum, infra-renal aorta and inferior vena cava. We believe that an angiographic study pre-operatively with embolization makes possible an analysis of the anastomosis and arterial supplement, making the ressecability of the tumor safer, although it seems the surgical intervention should have been done earlier.

Key words: Paraganglioma. Pheochromocytomas. Preoperative embolization.

\section{REFERÊNCIAS}

1. Sarmiento SA, Delgado FA, Álvarez MCB, Fabregat IP. Paraganglioma abdominal e cervical. Presentación de 1 caso. Rev Cubana Oncol. 1999; 15(3): 199-202.
2. Vassilopoulou-Selin R. Clinical outcome of 50 patients with malignant abdominal paragangliomas and malignant pheochomocytomas. Endocrine-Related Cancer. 1998;5(1):59-68. 
3. Boedeker CC, Ridder GJ, Schipper J. Paragangliomas of the head and neck: diagnosis and treatment. Fam Cancer. 2005; 4(1):55-6.

4. Rakovich G, Ferraro P, Therasse E, Duranceau A. Preoperative embolization in the management of a mediastinal Paraganglioma. Ann Thorac Surg. 2001; 72(2):601-3.

5. Liu D, Ma XC, Li BM, Zhang JG. Clinical study of preoperative angiography and embolization of hypervascular neoplasms in the oral and maxillofacial region. Oral Surg Oral Med Oral Pathol Oral Radiol Endod. 2006; 101(1):102-9. Epub 2005 Oct 12.
Recebido em 07/06/2006

Aceito para publicação em 25/07/2006

Conflito de interesse: nenhum

Fonte de financiamento: nenhuma

\section{Como citar esse artigo:}

Nakamura F, Silva RA, Santos VP, Razuk Filho A, Caffaro RA. Embolização pré-operatória no tratamento de paraganglioma abdominal: relato de caso Rev Col Bras Cir. [periódico na Internet] 2010; 37(2). Disponível em URL: http://www.scielo.br/rcbc

\section{Endereço para correspondência:}

Fernando Nakamura

E-mail:dr.nakamura@yahoo.com.br 